\title{
Reconstruction of chronic extensor hallucis longus tendon rupture using plantaris tendon graft
}

\author{
Ricardo Ummen de Almeida Tenório Villar ${ }^{(\mathbb{D})}$, Pedro Costa Benevides ${ }^{(\mathbb{D})}$, Caio Augusto de Souza Nery ${ }^{1,2}$ (D), Marcelo Pires Prado ${ }^{1 D}$, \\ José Felipe Marion Alloza' ${ }^{\mathbb{D}}$, Renato do Amaral Masagão ${ }^{(\mathbb{D})}$, Alexandre Leme Godoy-Santos ${ }^{1,3} \mathbb{( \mathbb { D }}$ \\ 1. Hospital Israelita Albert Einstein, São Paulo, SP, Brazil. \\ 2. Paulista School of Medicine, Universidade Federal de São Paulo, São Paulo, SP, Brazil. \\ 3. Professor Manlio Mario Marco Napoli Laboratory, Hospital das Clínicas, Faculdade de Medicina, Universidade de São Paulo, SP, Brazil.
}

\begin{abstract}
Chronic tendon ruptures can be difficult to manage as end-to-end repair can be challenging. In this scenario, reconstruction techniques with tendon grafts may be useful. The ideal tendon graft would be one that has similar biomechanical properties, low donor-site morbidity and length compatible with the tendon gap. We describe a safe technique for the reconstruction of a chronic extensor hallucis longus tendon rupture using a plantaris tendon graft.
\end{abstract}

Level of Evidence V, Therapeutic Studies; Expert Opinion.

Keywords: Hallux; Muscle, skeletal; Tendon injuries; Rupture.

\section{Introduction}

The treatment of neglected or chronic extensor hallucis longus (EHL) tendon ruptures can be challenging. Retraction or degeneration of the tendon stumps are not uncommon, and the presence of some degree of tendon retraction and/or degeneration often makes primary repair impossible, requiring the surgeon to master reconstruction techniques using grafts or tendon transfers. Reconstruction techniques using extensor digitorum longus (EDL)(1), semitendinosus, gracilis, peroneal longus, and plantaris tendons have been described in the literature, but there is no consensus on which technique is optimal.

Here we present a technical tip using a reconstruction technique with free plantaris tendon graft to treat a chronic EHL rupture in a 33-year-old patient. This is a safe procedure associated with less morbidity than other techniques.

\section{Clinical symptoms \& radiological findings}

A 33-year-old woman presented with the complaint of limited right hallux range of motion (ROM) for 5 months. She had dropped a sharp object on her right foot 4 years earlier. Physical examination showed a hallux flexus deformity with loss of active and passive dorsiflexion (Figure $1 \mathrm{~A}-\mathrm{H}$ ). Muscle strength was decreased in dorsiflexion to the right hallux. Foot and ankle radiographic findings were normal. Magnetic resonance imaging $(\mathrm{MRI})$ revealed an $\mathrm{EHL}$ rupture with the proximal stump at the level of the head of the talus (Figure 2A-G). The proximal and distal tendon ends were irregular and tortuous with a heterogenous sign. MRI also showed a dorsal talar osteophyte. Dynamic ultrasound confirmed the $\mathrm{EHL}$ rupture, showing the proximal stump at the level of the talonavicular joint and the distal stump at the mid-diaphysis of the first metatarsal. During the dynamic maneuver, we
Study performed at the Hospital Albert Einstein, São Paulo, SP, Brazil.

Correspondence: Ricardo Ummen de Almeida Tenório Villar. Av. Albert Einstein, 627, Jardim Leonor-05652-900, São Paulo SP, Brazil.E-mail: ricardovillar_@hotmail.com. Conflicts of Interest: none. Source of funding: none. Date received: July 09, 2021. Date accepted: July 12, 2021. Online: August 31, 2021.
How to cite this article: Villar RUAT, Benevides PC,

Nery CAS, Prado MP, Alloza JFM, Masagão RA,

et al. Reconstruction of chronic extensor hallucis

longus tendon rupture using plantaris tendon graft. J Foot Ankle. 2021;15(2):179-82. 

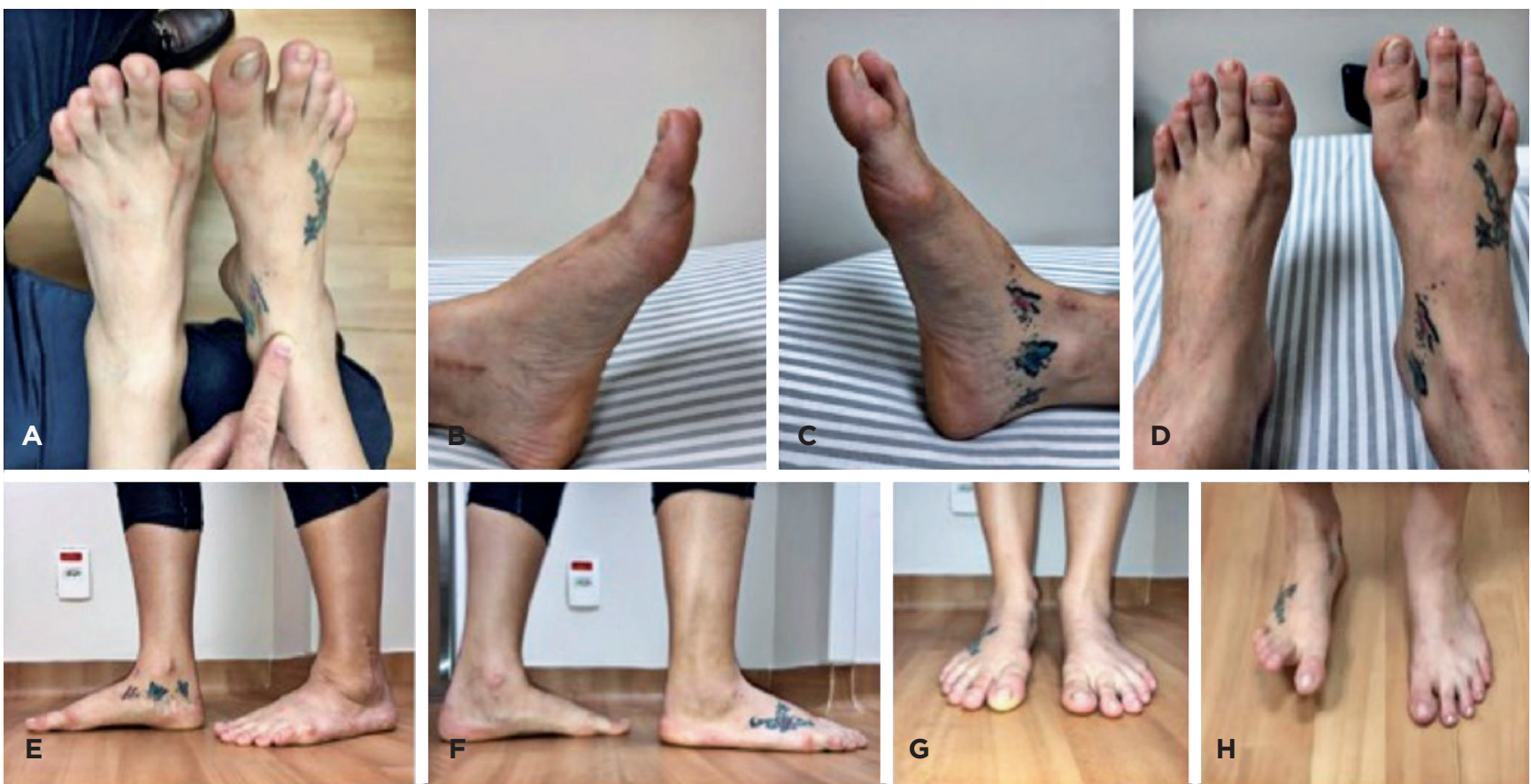

Figure 1. Preoperative clinical photographs.
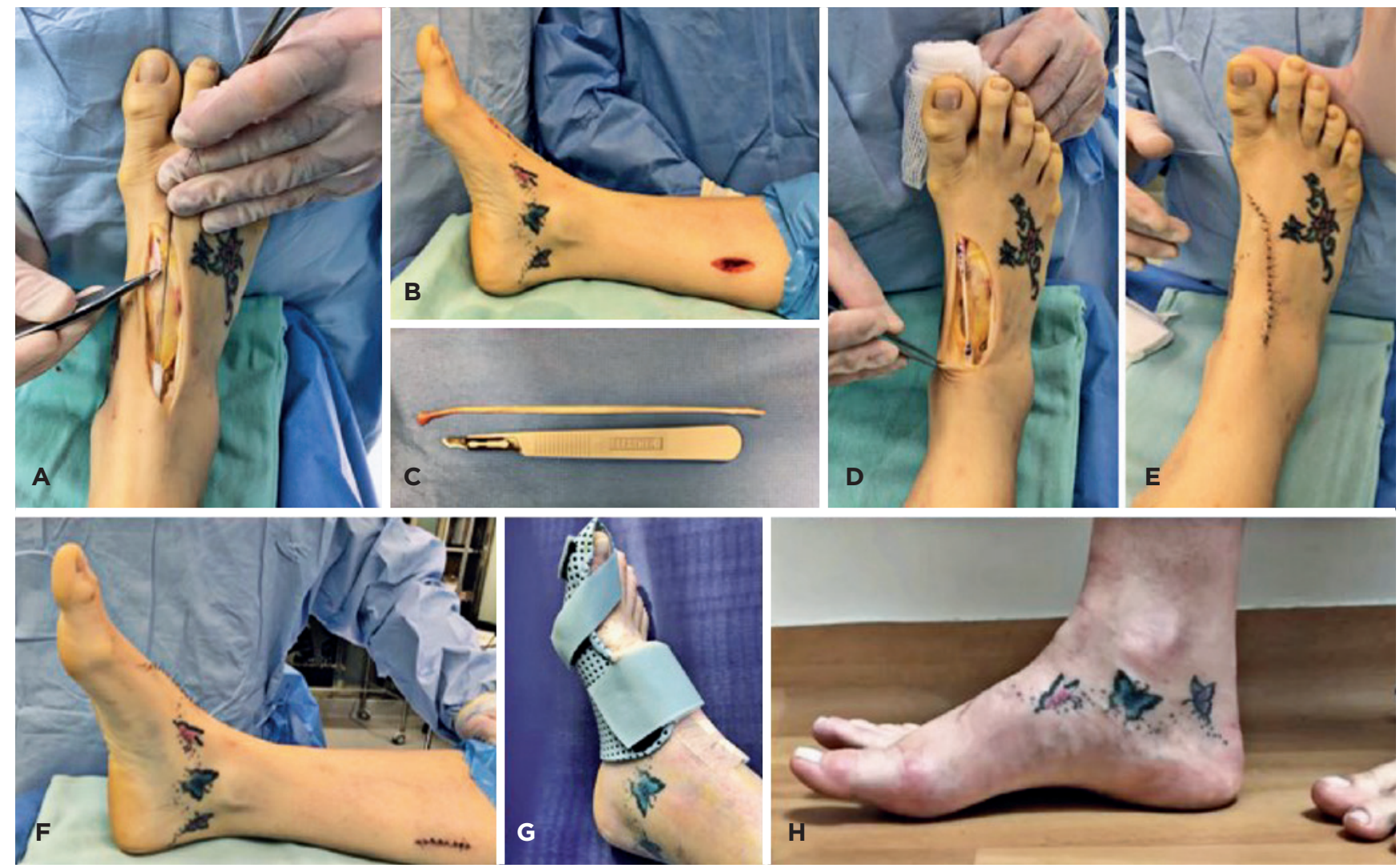

Figure 2. Intraoperative and postoperative photographs. 
observed a synchronic motion of the two stumps-they were apparently still partially connected by a thin $(1 \mathrm{~mm})$ fibrous scar tissue. Electromyography of the right lower limb revealed abnormalities in the deep peroneal nerve at the extensor retinaculum suggestive of compression. We performed operative treatment.

\section{Technical tips}

With the patient in the supine position under spinal anesthesia with a pneumatic tourniquet applied to the thigh, we made a dorsal incision along the course of the EHL tendon and identified the distal stump at the level of the neck of the first metatarsal. We extended the incision proximal to the level of the extensor retinaculum, where we identified the proximal stump. We debrided each end of both stumps and noted a large tendon defect (Figure 3A). We identified some osteophytes at the talonavicular joint and debrided them as well. In the middle third of the lower leg, we made a $3-\mathrm{cm}$ longitudinal incision just medial to the tibia (Figure 3B). We then made an incision in the fascia, identified the plantaris tendon at its descending course and performed a proximal tenotomy. We harvested the plantaris tendon by advancing a stripper down to its calcaneal insertion, thus obtaining a $14-\mathrm{cm}$ graft (Figure $3 \mathrm{C}$ ). We anastomosed the graft to the proximal and distal stumps using 3-O Vicryl ${ }^{\circledR}$ with a latero-lateral technique (Figure 3D). We closed the wounds in layers (Figure 3E) and applied a negative-pressure dressing $\left(\mathrm{PICO}^{\circledR}\right)$. We confirmed that the neutral position of the hallux was maintained after the repair (Figure 3F) and applied a short-leg cast. We removed the stitches after 2 weeks, when the patient began physical therapy with ROM exercises (except for plantar flexion). We then placed a moldable thermoplastic splint to hold the hallux in extension postoperatively (Figure 3G). We kept the patient non-weight-bearing for 6 weeks after surgery. At 3 months, the patient resumed her daily activities with no restrictions.

The correct position of the hallux was maintained after rehabilitation until the last visit (Figure $2 \mathrm{H}$ ).

\section{Discussion}

Extensor tendon injuries account for $1 \%$ of all foot injuries ${ }^{(1,2)}$. The surgical treatment of neglected extensor tendon ruptures ( $>6$ weeks) often requires the use of grafts due to tendon stump fibrosis and retraction. Al-Qattan proposed an anatomic classification based on the zone of injury ${ }^{(1,3)}$ :

Zone 1: at the insertion site on the distal phalanx.

Zone 2: the area between zones 1 and 3 .

Zone 3: over the first metatarsophalangeal joint.
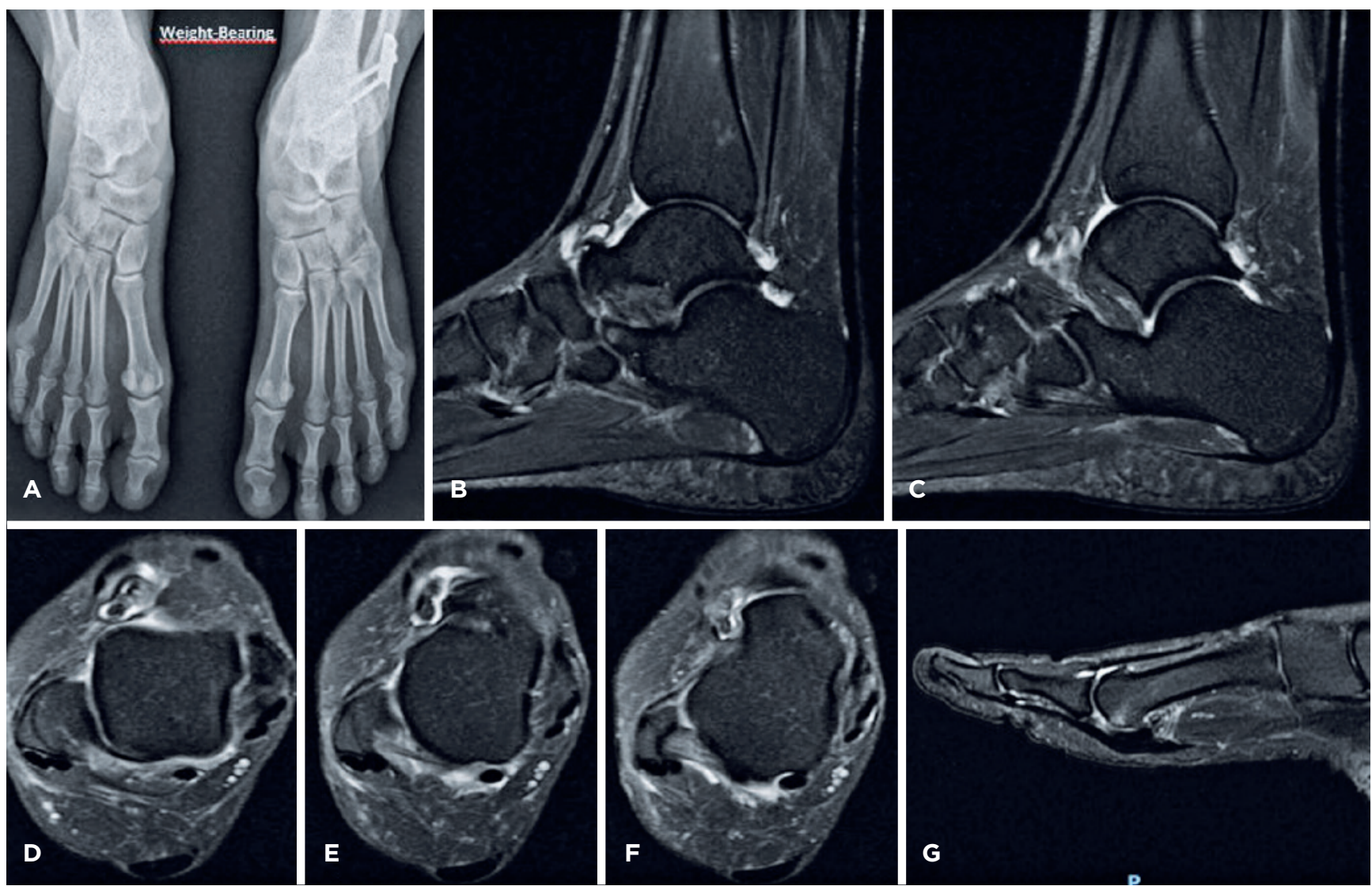

Figure 3. Radiographs and magnetic resonance images. 
Zone 4: on the dorsum of the foot between zones 3 and 5.

Zone 5: laceration of the tendon beneath the extensor retinaculum.

Zone 6: in the lower leg proximal to the extensor retinaculum.

For late reconstruction of the $\mathrm{EHL}$ tendon requiring lengthening, techniques have been described using EDL(1), semitendinosus, gracilis, peroneal longus, plantaris tendon, and accessory extensor of the EHL tendon(4), in addition to the possibility of using an allograft from a tissue bank.

The use of grafts has been associated with donor-site morbidity, such as pain and loss of function. Using an allograft is an option to avoid donor-site morbidity; however, allografts are not widely available and require special infrastructure for collection, sterilization, and transportation.

Surgical results can be classified according to the grading system proposed by Lipscomb and Kelly apud Al-Qattan(3), as follows: good, the patient has normal ROM of the hallux (compared to contralateral) and no pain; fair, the patient has painless, active hallux extension and no tripping over the hallux when walking barefoot; and poor, the patient has no active hallux extension, tripping over the hallux when walking barefoot, or persistent pain.
We described here a technique with low patient morbidity, since the plantaris muscle is of secondary importance in biomechanics and is present in more than $90 \%$ of individuals ${ }^{(5)}$. The plantaris is one of the muscles of the superficial posterior compartment of the leg, originating from the lateral femoral condyle and inserting into the medial side of the calcaneus in most patients. It acts as an ankle plantar flexor and is a weak knee flexor. Savita et al.(6), in a study of 48 legs ( 24 patients), reported that the plantaris tendon length ranged from 34 to $38 \mathrm{~cm}$ in $66.5 \%$ of cases, being absent in $12.5 \%$.

Yammine et al. ${ }^{(5)}$, in a meta-analysis conducted in 2019 that compiled the results of 41 studies with a total of 10062 legs, also showed that plantaris tendon grafts had satisfactory length $(30.63 \pm 5.87 \mathrm{~cm})$ and thickness $(3.68 \pm 1.37 \mathrm{~mm})$. These morphologic features are important because this graft allows us to perform several surgical techniques, including double grafts for greater resistance.

In the case reported here, the plantaris muscle, as it is a vestigial structure with a long tendon, provided a safe tendon graft option with low associated morbidity for the reconstruction of chronic EHL ruptures with large gaps between tendon stumps.

Authors' contributions: Each author contributed individually and significantly to the development of this article: RUATV *(https://orcid.org/OOOO-OOO24563-5726) Conceived and planned the activities that led to the study, participated in the review process, data collection; PCB *(https://orcid.org/OOOO0002-4209-0564) Conceived and planned the activities that led to the study, participated in the review process, clinical examination; CASN *(https://orcid. org/0000-0002-9286-1750) Approved the final version, participated in the review process; MPP *(https://orcid.org/0000-0003-3812-9320) Approved the final version, participated in the review process; JFMA *(https://orcid.org/0000-0002-7664-2064) Data collection, formatting of the article; RAM *(https:// orcid.org/0000-0002-7830-8318) Data collection, formatting of the article; ALGS *(https://orcid.org/0000-0002-6672-1869) Performed the surgeries, conceived and planned the activities that led to the study, clinical examination. All authors read and approved the final manuscript. *ORCID (Open Researcher and Contributor ID) (iD).

\section{References}

1. Wong JC, Daniel JN, Raikin SM. Repair of acute extensor hallucis longus tendon injuries: a retrospective review. Foot Ankle Spec. 2014;7(1):45-51.

2. Jaffe D. Restoration of Extension of the Great Toe After an Untreated, Retracted Extensor Hallucis Longus Rupture: A Case Report. JBJS Case Connect. 2019;9(4):e0348.

3. Al-Qattan MM. Surgical treatment and results in 17 cases of open lacerations of the extensor hallucis longus tendon. J Plast Reconstr Aesthet Surg. 2007;60(4):360-7.
4. Kurashige T. Chronic extensor hallucis longus tendon rupture treated with double-bundle autograft of extensor hallucis capsularis: A case report. SAGE Open Med Case Rep. 2019;7:2050313X19841962.

5. Yammine K, Saghie S, Assi C. A Meta-Analysis of the Surgical Availability and Morphology of the Plantaris Tendon. J Hand Surg Asian Pac. 2019;24(2):208-18.

6. Savita K, Tuli A, Agarwal S. Morphology of plantaris longus with special reference to its clinical importance. Int J Anat Res 2019;7(4.2):7101-4. 